\section{Archivos \\ de Cardiología de México}

\title{
Disección aórtica posterior a cirugía de Bentall y de Bono
}

\author{
Aortic dissection after Bentall and Bono surgery \\ Mario A. Ornelas-Casillas y Mario R. García-Arias* \\ Departamento de Cardiología, Instituto Nacional de Cardiología "Ignacio Chávez", Ciudad de México, México
}

Hombre de 41 años con antecedente de reparación quirúrgica mediante procedimiento de Bentall y De

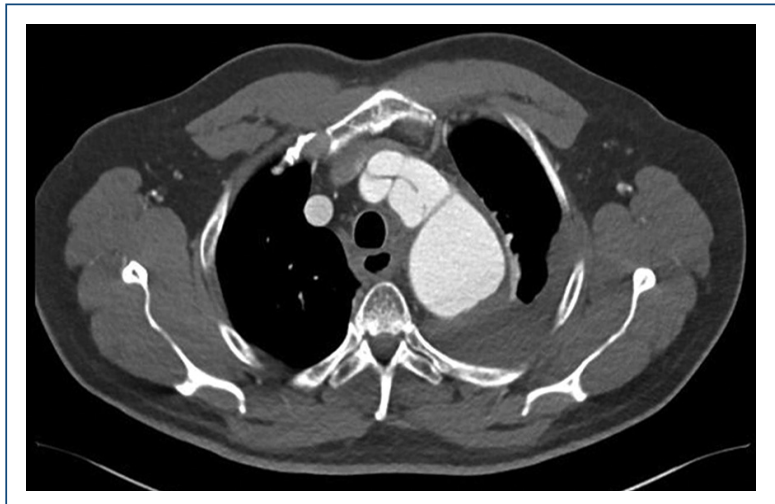

Figura 1. TAC: corte axial en fase arterial a nivel del arco aórtico.

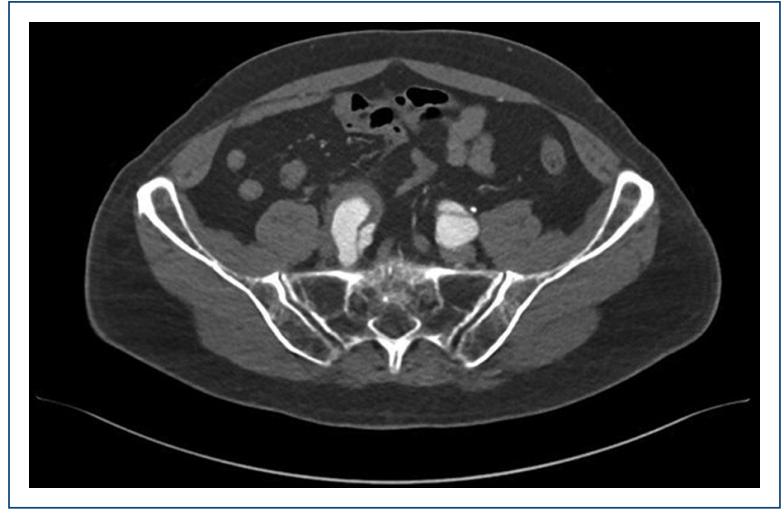

Figura 2. TAC: corte axial en fase arterial a nivel de la bifurcación aortoilíaca.
Bono. Acudió a urgencias por debilidad y claudicación de extremidades inferiores en relación con hipotensión. Dímero D de $0.901 \mu \mathrm{g} / \mathrm{ml}$. La TAC contrastada en fase arterial y venosa mostró los hallazgos observados en las Figuras 1 y 2: disección aórtica Stanford A, DeBakey III; luego del injerto aórtico se identifica colgajo (flap) de disección (Fig. 3 y 4).

Las ramas viscerales y los vasos supraaórticos emergen de la luz verdadera. Después de la emergencia de la arteria subclavia izquierda se encuentra un aneurisma fusiforme de extensión caudal.

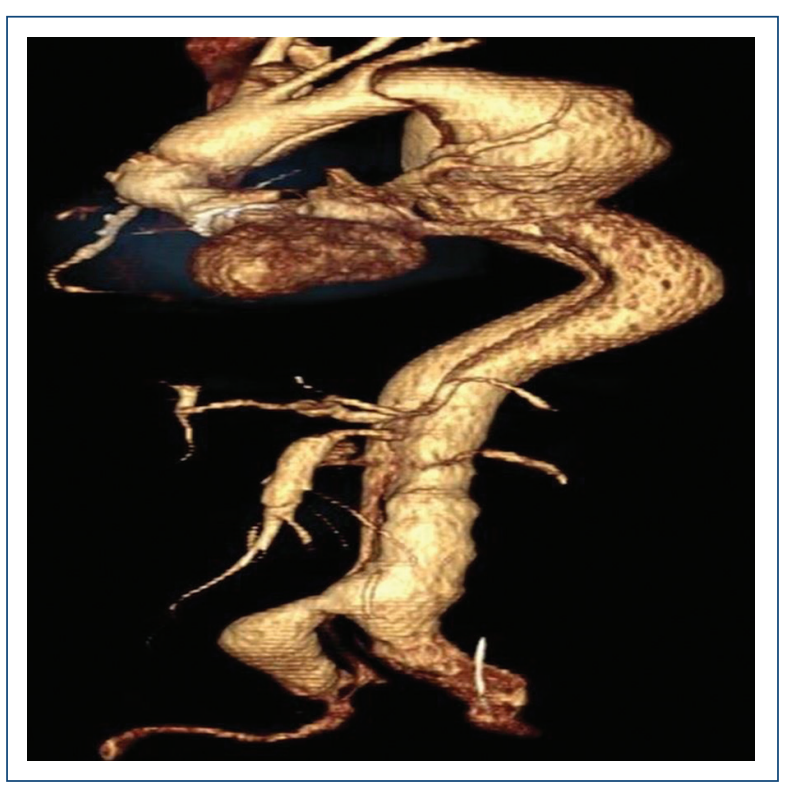

Figura 3. Reconstrucción tomográfica tridimensional de la aorta.

\section{Correspondencia:}

*Mario R. García-Arias

E-mail: mario.aris7@gmail.com
Disponible en internet: 27-07-2021 Arch Cardiol Mex. 2022;92(1):116-117 www.archivoscardiologia.com

1405-9940 / @ 2021 Instituto Nacional de Cardiología Ignacio Chávez. Publicado por Permanyer. Este es un artículo open access bajo la licencia CC BY-NC-ND (http://creativecommons.org/licenses/by-nc-nd/4.0/). 


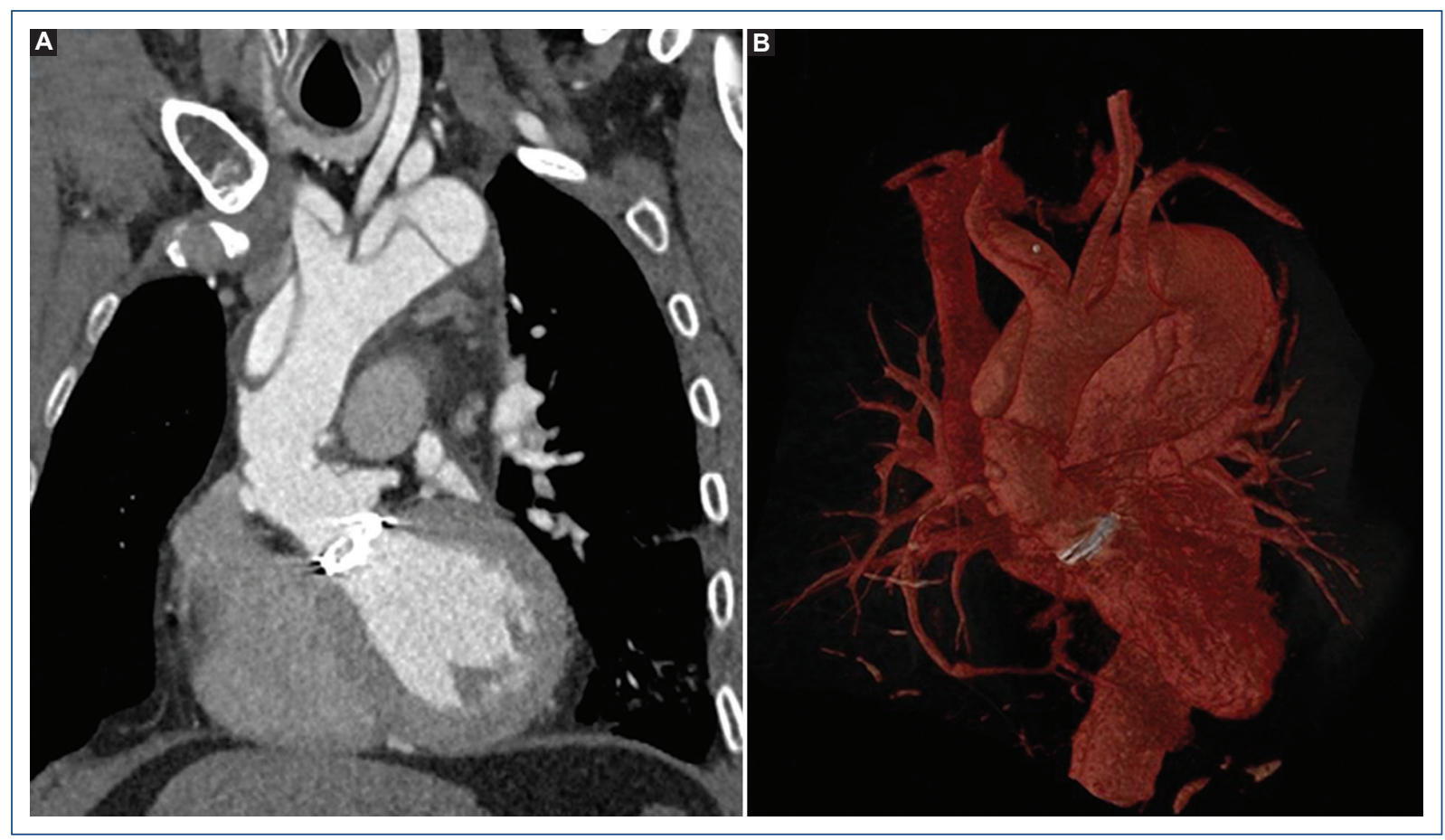

Figura 4. A: Corte coronal: se observa la parte distal de la prótesis de Bentall-De Bono y el inicio del colgajo de disección aórtico. B: Reconstrucción tridimensional de la imagen A.

\section{Conclusiones}

La incidencia de disección aórtica posterior a una cirugía cardíaca es del $15 \%{ }^{1}$. Sólo el $3.6 \%$ de los pacientes sometidos a procedimiento de Bentall y De Bono requiere una nueva intervención ${ }^{2}$. Este caso clínico tiene como propósito notificar una complicación relativamente infrecuente, si bien con un índice elevado de mortalidad.

\section{Agradecimientos}

Los autores expresan su agradecimiento al Instituto Nacional de Cardiología "Ignacio Chávez" por el apoyo y la colaboración para la elaboración de este documento.

\section{Financiamiento}

Esta investigación no ha recibido ninguna beca específica de agencias de los sectores público, comercial, o sin ánimo de lucro.

\section{Conflicto de intereses}

Ninguno.

\section{Responsabilidades éticas}

Protección de personas y animales. Los autores declaran que para esta investigación no se han realizado experimentos en seres humanos ni en animales.

Confidencialidad de los datos. Los autores declaran que han seguido los protocolos de su centro de trabajo sobre la publicación de datos de pacientes.

Derecho a la privacidad y consentimiento informado. Los autores declaran que en este artículo no aparecen datos de pacientes.

\section{Bibliografía}

1. Nienaber CA, Clough RE, Sakalihasan N, Suzuki T, Gibbs R, Mussa F, et al. Aortic dissection. Nat Rev Dis Primers. 2016 Jul 21;2:16053.

2. Joo HC, Chang BC, Youn YN, Yoo KJ, Lee S. Clinical experience with the Bentall procedure: 28 years. Yonsei Med J. 2012;53(5):915-23. 